and quantity of parent-teen communication about safe driving. Evaluations have shown both interventions to be effective independently, but no studies have examined parent-teen interaction related to in-vehicle feedback systems. 153 parent-teen dyads were recruited through local high schools and randomised to one of three groups: control; in-vehicle video feedback; and feedback with STS.

Preliminary Results During baseline (4 weeks), groups averaged between 22 and 27 driving errors per week. The STS plus video group reduced their average driving errors to 8 in the first month (a 64\% reduction) and to five or less in the second through fourth months $(-77 \%)$. The video only group had a slight reduction of 27 to $23(-15 \%)$ driving errors in the first month, then reduced to ten or less for months two through four $(-63 \%)$. The control group did not show any decrease in driving errors. Proportionate hazards models indicate that the STS group had a significantly faster reduction in driving errors, and both intervention groups had significant reductions by the fourth month.

Conclusions In-vehicle video feedback systems effectively reduce driving errors, and the effectiveness is significantly improved when paired with a parent-focused communication program.

\section{YOUNG DRIVER CRASH TYPES AND LIFETIME CARE COSTS BY POSTED SPEED LIMIT}

${ }^{1}$ Samantha Buckis, ${ }^{1}$ Mike G Lenné, ${ }^{1}$ Amanda Stephens, ${ }^{2} \mathrm{C}$ Raymond Bingham and ${ }^{1}$ Michael Fitzharris. 'Accident Research Centre, Monash University, Melbourne, Australia; ${ }^{2}$ UMTRI, University of Michigan, Ann Arbour, USA

\subsection{6/injuryprev-2016-042156.330}

Background Speeding is a key issue for all drivers as it influences both crash risk and crash severity. Young drivers, particularly males, are more likely to engage in speeding behaviour and speeding is a common contributing factor in young driver crashes. Greater understanding of the impact of speed and speeding on young driver crash risk and crash outcomes is required to inform the development, prioritisation and implementation of evidence-based safety interventions.

Methods In Victoria, Australia, the Transport Accident Commission (TAC) operates as the sole, statutory compensation $\left(3^{\text {rd }}\right.$ party insurance) scheme. The TAC has legislated responsibility for road safety and the care of persons injured in road crashes, irrespective of fault. TAC claims data for the period 2005-2013 was used to analyse the differences in crash types and injury outcomes, including cost, by posted speed limit (30, 40, 50, 60, 70, $75,80,90,100$ and $110 \mathrm{~km} / \mathrm{h})$. The mean and overall lifetime care costs were analysed by speed limit. Speed limit was also analysed by age, gender, location, time of day and crash type. Young drivers were split into two groups for comparison 18-21 and 2225.

Results Over the 9-year period, 16,794 claims were lodged to the TAC for killed or injured drivers 18-25 years of age (young drivers). The total lifetime care cost for all young drivers killed or injured in a crash was estimated to be \$AUD634 million. The total lifetime care cost for young male drivers was estimated to be \$AUD470 million compared to \$AUD163 million for young female drivers. 18 to 21 year old drivers accounted for \$AUD356 million compared to \$AUD279 million for 22 to 25 year old drivers. When the posted speed limit is considered, the total cost of killed or injured young drivers who crashed in $60 \mathrm{~km} / \mathrm{h}$ zones $(\mathrm{N}=3914)$ was estimated to be \$AUD70 million $(M=$ \$AUD
17,807), compared to \$AUD208 million ( $M=$ \$AUD 65,580$)$ in $100 \mathrm{~km} / \mathrm{h}$ zones $(\mathrm{N}=3173)$.

Conclusions Road trauma not only has an enormous impact on the lives of those involved, their families and friends, it also has a significant economic burden on our communities. This research demonstrates that speed and male gender play key roles in crash outcomes, particularly lifetime care costs, and should be essential considerations for safety interventions.

\section{WHY RESEARCH IS INDISPENSABLE TO SMART PUBLIC POLICY}

Anthony Green. Safe Kids Worldwide

\subsection{6/injuryprev-2016-042156.331}

Background Laws are essential in all efforts to save young lives. It is important to reinforce the indispensable role evidence plays in winning an advocacy effort, just as evidence is the foundation for all aspects of public safety campaigns, messaging and education.

Description of the problem In 2013, 2,163 kids 16-19 were killed in U.S. car crashes. Drivers aged 16-19 are nearly three times more likely to be in a fatal car crash than those aged 20 and older. The risk is greater because teens are less likely to recognise road dangers than older drivers and more likely to exceed speed limits. Of kids 13-20 dying in motor vehicle crashes, only $56 \%$ were wearing seat belts.

Results There is a body of evidence that stronger Graduated Driver License (GDL) laws save lives as demonstrated in the U.S., and also in Canada, Sweden and New Zealand. Since Illinois passed a tougher GDL law there's been a 57\% reduction in teen motor crashes. Connecticut has one of the most stringent GDL laws in the U.S., and in 2014, not a single 16- or 17- year old passenger was killed in a crash involving a driver in the same age group. In addition, evidence based online applications can predict how many lives are saved through various GDL interventions. As an example, a "calculator" created by the respected Insurance Institute for Highway Safety predicts that if Arkansas raised its permit age to 16 and limited night driving, there could be a $26 \%$ reduction in fatal crashes. Safe Kids has cited the calculator in a successful advocacy effort in the U.S. Senate and it was used in an effort in New Jersey. The combination of real time data and modelling tools from a credible research organisation are powerful weapons to influence child safety policy change.

Conclusions Advocacy campaigns require a foundation of data and evidence, and the research function should be part of the planning process in developing a campaign.

\section{INNOVATIVE STRATEGIES TO REDUCE TRAFFIC RELATED INJURIES AND DEATHS IN YOUTH}

${ }^{1}$ Joanne Banfield, ${ }^{2}$ Donald Redelmeier. 'Sunnybrook Health Sciences Centre, Canada; ${ }^{2}$ University of Toronto, Canada

\subsection{6/injuryprev-2016-042156.332}

Background Road traffic injuries are the leading cause of death among young people, aged 15-29 years. Studies have shown young drivers are more likely to underestimate the probability of specific risks caused by traffic situations, as well as to overestimate their own driving skills making them more vulnerable to trauma. The P.A.R.T.Y. (Prevent Alcohol and Risk-Related Trauma 
in Youth) Program, developed in 1986 is a one day, in hospital injury awareness and prevention program for youth aged 15 and older. The goal is to provide young people with information about trauma that will enable them to recognise their injury risks, make prevention-oriented choices and adopt behaviours that minimise unnecessary risks through vivid clinical reality.

Methods Several research studies have been undertaken to determine effectiveness and changes in attitudinal risk behaviour from youth attending the P.A.R.T.Y. A ten-year longitudinal study was conducted to determine whether students who attended P.A.R.T. Y. had a reduction in injuries compared with a matched control group of students based on age, gender and geographic area who did not attend the program.

Students follow the course of injury from occurrence through transport, treatment, rehabilitation and community re-integration phases.

Additionally by augmenting a didactic format through a technologically innovative approach including but not limited to vivid clinical reality, social media, interactive websites and simulators we see attitudinal and behavioural changes.

Results The 10 year longitudinal study showed P.A.R.T.Y. participants had a lower incidence of traumatic injuries than a control group of non-P.A.R.T.Y. participants of the same age, gender, residential area, and initial year in database, during the 10 -year study.

Conclusions Research-driven, psycho-social theories of behaviour and technologically innovative approaches have proven it is possible to influence behaviour through the delivery of welldesigned and well-executed road safety strategies, programs and campaigns. Providing students with real-life education to depict the vivid clinical reality of injuries was shown to be a compelling and effective method of education.

\section{Technology - Solutions and Applications for Safety}

\section{Parallel Tue 3.3}

\section{TRANSLATING EVIDENCE INTO PRACTICE; DEVELOPMENT OF THE KNOW INJURY KNOWLEDGE HUB}

Venessa Wells, Rachel Meade, Roisin Sweeny, Marc Zen, Megan De Piazz, Jennifer Affric. Injury Control Council of Western Australia, Australia

\subsection{6/injuryprev-2016-042156.333}

Background Knowledge exchange portals are effective tools to exchange information, resources and connect practitioners. It is well established that public health policy and program design should be informed by evidence. Evidence informed practice utilises the best available knowledge of what works and why in the design and delivery of policies and programs. Accessing and exchanging knowledge between practitioners is essential to evidence selection.

Problem Formative evaluation identified the need for a platform for injury prevention practitioners to access and share evidence across Western Australia (WA). We present an overview of the development, implementation and evaluation of the Know Injury Knowledge Hub (KH).

Results The Know Injury team at the Injury Control Council of Western Australia (ICCWA) developed the KH with the support of the WA Department of Health. The purpose was to provide information, tools and training to enhance the capacity of injury prevention practitioners to deliver, evidence informed activities.

The hub is built upon three pillars; Know, Learn, Connect and is underpinned by a public health approach to injury prevention. It builds practitioners' knowledge about injury topics, providing an understanding of the extent of the problem, determinants, effective strategies, current initiatives and key stakeholders in WA.

It focuses on increasing practitioner skills in relation to health promotion planning, implementation and evaluation and provides opportunities for practitioners, particularly those who are geographically isolated to initiate and build networks and partnerships with other practitioners.

Conclusion Driven by principles of sharing and knowledge, the hub utilises discussion boards, blogs, networking opportunities and online chat to encourage knowledge exchange. The $\mathrm{KH}$ is a single point of access for people with an interest in injury prevention and community safety and provides support for the delivery of evidence informed initiatives.

\section{USING SOCIAL MEDIA TO IMPROVE PARTICIPATION IN A SMOKE ALARM DISTRIBUTION AND INSTALLATION PROGRAM}

Shannon Frattaroli, Eric Schulman, Eileen McDonald, Wendy Shields, Elise Omaki, Vanya Jones. The Johns Hopkins Centre for Injury Research and Policy, USA

\subsection{6/injuryprev-2016-042156.334}

Background In 2014, residential fires caused 2745 civilian deaths in the United States (US) and almost 12,000 injuries. Smoke alarms are an effective intervention for reducing residential fire deaths. Innovative strategies are needed to improve the prevalence of working smoke alarms in homes. We used Facebook advertising to encourage participation in a free smoke alarm installation program in one US city.

Methods As part of a larger evaluation of an enhancement to a smoke alarm distribution program, we developed three ads that ran on Facebook throughout October 2014 with a US\$100 daily budget. The ads included three different images and a motivational message. The ads appeared on the news feeds and mobile news feeds of adult Baltimore City residents.

Results The three ads delivered to desktop and mobile device news feeds resulted in 465,666 impressions and reached 130,264 discrete users (43\% of those 300,000 users estimated to be eligible). Most users viewed the ads on a mobile device (99\%). Of the Facebook users reached, 4,367 different users (3.4\% unique click-through rate) clicked the ad. Some users clicked through more than once, resulting in 6,075 total clicks. Mobile devices yielded higher click-through rates than desktops $(1.31 \%$ and $0.36 \%$ respectively). Of the 4,367 unique clicks, nearly all $(4,357)$ came from mobile devices.

The cost per impression was less than one cent. We spent two cents to reach each user, and 70 cents on each unique user who clicked on the ad. For those 25 participants who requested a smoke alarm installation and cited Facebook as their information source about the program, we spent \$124 to achieve that target behaviour.

Conclusions Facebook advertisements are a novel approach to promoting smoke alarms and appear to be effective in exposing individuals to a service program. However, converting Facebook 